\title{
OFFLINE NAVIGATION SYSTEM FOR MOBILE DEVICES
}

\author{
Prithumit Deb, Nitin Singh, Saket Kumar, Nitish Rai \\ School of Information Technology and Engineering \\ VIT University, Vellore, Tamil Nadu-632014 \\ prithumitls@gmail.com, nitin.biz.singh@gmail.com, \\ saki007ster@gmail.com,nitishrai2@gmail.com
}

\&

\author{
Dr. P. A. S. Naidu \\ D. B. Science College, Gondia (MS),India \\ anjannaidu@indiatimes.com
}

\&

N.Ch.Sriman Narayana Iyengar

School of Computing Science and Engineering

VIT University, Vellore, Tamil Nadu-632014 nchsniyengar48@gmail.com

\section{ABSTRACT}

The theme of the software that we have been developing is a combination of Mobile Solutions and Leveraging Geo data and Maps to organize the world's information and to make it universally accessible and useful to a wider and better extent. Our project is a mobile application that allows the user to download and install street maps of a particular city, state or country depending on his current needs from a site. The downloaded maps can be browsed offline by the user and the user can download new maps or update the existing ones whenever he feels the need to do so. The downloaded maps will be dynamic giving the user the feel as if he is browsing the maps online. The user's current location will be shown on the map using the cell tower info that is provided by all the cell network providers thus making the application usage completely independent of internet service. The downloaded maps will not be like some image of a street map but like a fully functional and active map that allows the user to search for a particular place like some mall, or some spot for tourist interest by just speaking the name of the place which will be processed by the speech recognition system which is a great feature of our application specially useful for people who don't know how to read or write in English. The result produced by the application will be a shortest by road route to the place which will be highlighted on the map ,the distance and the estimate cost of reaching there by a cab or a bus service, any transportation means like a bus stand or a taxi stand in the close vicinity of the users current location will be highlighted in the map. The 
cursor on the map indicating the user's current location will move along with the user as he travels thus providing him real time information of his location.

\section{INTRODUCTION}

The motivation behind our project is in complete synchronisation with the theme that is to organize the world's information and make it universally accessible and useful. The application developed will combine mobile solutions and geo-mapping to provide critical informational active maps to tourists, travellers, hikers or any person trying to seek information about some place that is unknown to him. The application will be capable of reducing the tourist's frustration and stress to a great extent as he will have all the information he needs on the palm of his hands that is on his mobile device. The best part is that the information will be there with him all the time without requiring him to have any access to any sort of internet service, all he needs to do is to download and install the maps of the place/places he will be visiting before leaving and the application will take care of the rest. This service will work in areas having very week cell networks like rural or remote places as all it needs is a very minimal gps/cdma connection to get the cell info display for locating the user's current location.

As most of the data that is the maps and the information related to developing mapping applications is already available so the development of the application won't cost much as the only work that needs to be done is organizing the available information properly. This application will clearly increase the accessibility of the information as it allows mapping services to be used by people who don't have $24 \times 7$ access to internet or in countries where the internet services provided by the cellular service providers is bad or unreliable .The application will surely be of great use to travellers and people visiting rural or remote areas and in that case to the rural population itself. The cherry on the cake is that the quality and content of the service would be same as if the user was accessing the maps online giving him total customer satisfaction.

\section{RELATED WORKS}

Leveraging of Geo data and Mapping Technologies in Mobile devices has been existent for quite a few years now. Most of the mapping technologies we have now are Online or Global Positioning System (GPS) based, like the Nokia Maps and Google Maps which are the 
market leaders in mapping technologies and mobile navigation system now. Converting Navigation to Offline and making it more user-friendly and accessible to a wider range of people with limited configuration mobile devices (not only the high-end GPS enabled phones) is rarely witnessed and this is the main motivation behind our project, i.e. developing an user-friendly offline navigation system for mobile devices with wider accessibility and limited configuration needs.

\section{USE CASES}

Generally, anyone who travels or loves to visit new places can use our product. Mainly tourists are the people who can use it, as it locates nearby tourists spots by locating their position and gives information how to get there. It even can be used by the local people who need the basic information like distance between two places, fair of the taxi from one place to other. Its offline mode capability increases its demand in the section of the people who cannot access internet easily. Detailed description about the well known places and nearby spots to those places is also a very important feature which will enable the people to find places and about them. This application can be easily installed on any java enabled multimedia phone and its map has to be downloaded from the site of it .Download the map of whichever city, area or country you want and save it in its data folder. And start using it. It has a voice interface that acts on the voice commands given to it, you just speak the place you want to search and you will have it on the screen with the required details.

\section{KEY FEATURES}

$>$ Use of Voice recognition technology:-

A Speaker independent voice recognition system is used for this software in which enables the user to find a particular location in the map using speech. This unique feature eliminates the process of typing the location name, reluctance in remembering spellings which leads to errors. Also this increases the simplicity of usage, and makes its easy even for the illiterates and disabled persons to use this software.

\section{$>$ Offline mapping technology:-}

This software is an offline application. Compared to the other online-mapping software, this software doesn't need an internet connection for finding out places nor does it needs a GPS technology or other connectivities mainly used by online mapping software. 


\section{Increased Geographical Information accessibility:-}

Relatively large amount of geographical information will be available to the user through this software such as:

- User's present geographic location.

- Distance between the user's source and destination places.

- Shortest and easiest path available to the user.

- Information about nearest tourist places and the important areas in a particular place which will serve as a guide to travellers.

\section{$>$ Simplicity in Usage and Access:-}

This is one of the main features of this geo-mapping software. The use of voice technology combined with a mobile device system will provide the general users with great simplicity and ease of use. Even technologically weak users can use this software as the commands are given through voice and downloading this software is simple.

\section{$>$ Portability:-}

As this is software is built by using java applications for mobile devices and makes use of offline mapping technology, it offers great portability. Users can use it in their cellphones without having to access the internet. Travellers and tourists will be the most benefited ones.

\section{Ease of Updation and download:-}

This software can be easily updated against a geographic change because the software will be made for particular areas in pieces, so any change in that particular area is easy to modify. Also downloading will be easy as the user needs to only download the software for his particular required area. The updated versions will be readily available in the website which can be easily downloaded or purchased.

\section{Secure and Reliable application:-}

As it is offline mobile device software, it is naturally secure from the threats of the internet (viruses and Trojans) and the high level of details provided for the required area and its ease of accessibility makes it very reliable.

\section{SYSTEM DESIGN OVERVIEW}

The Design Procedure of our mobile application which is a offline geo-mapping software which makes use of voice recognition technology for input is described below. The system 
consists of the following components which are discussed in detail below with all their operations.

\section{User Input Interface:-}

Our software will use a user friendly voice recognition technology apart from the text input interface through which the user can enter the name of the area to be searched in the mapping software.

\section{Voice recognition input system}

This software will use a speaker independent voice recognition system through which the user can input the area name to be searched. The system will convert the word spoken by the user to the text form and will use it for searching the required destination. Let us see briefly how this system will be designed:-

a) Firstly, we will use the hashing technique to store the names of all the places that can be located in the map in a hash table. By using a hash function we will get a unique key for each place on the map. This key will be used for searching and referencing.

b) Next are the processes of converting the speech to data. The analog speech signal picked up by the mobile sound input system will be converted into digital its appropriate digital text.

c) We will first filter the voice input wave entered by the user using different techniques so that we get a good quality voice wave for processing which will contain the least disturbance. The processes used in voice filtering are:-

a. Removal of unwanted noise and background disturbances.

b. Normalization.

c. Digital sampling.

d) Phoneme Extraction. Next the voice input wave is divided into small segments as short as a few hundredths of a second or even thousandth known as phonemes. English uses about 40 phonemes to convey the 500,000 or so words it contains, making them a relatively good data item for speech engines to work. Next the extracted phonemes are matched with their digital format and using this, the "phonetic word" is constructed from the phonemes.

e) Next we convert the phonetic word into the spoken word using a "phoneme to English translation" dictionary. Thus we will get our required word in text form from the user's spoken voice. 
f) Finally we will generate the "key" again for this text word using the same hash function used above and match it with the key in the hash table to locate the required place on the grid map. Thus the exact grid for that particular place can be referenced.

\section{$>$ Text based input system}

Apart from the voice input system, the user can also enter the name of the place to be searched using text (with correct spelling). The text search system is simple and will make use of the same hashing technique as mentioned above in step a) and will be directly searched for the key as mentioned above in step f).

Using the system of voice recognition input, will be simpler for the users and will be very beneficial for the illiterate persons and persons with disability.

\section{Databases:-}

Databases comprises of maps, names of all possible places mentioned on the map and details of those places dealing in total area of the place, other important places around the searched place and along the roads from source to destination.

The maps we use here are divided into grids according to defined scale. These scales are dependent on the area of the region originally shown by the map. As we try to zoom in a particular grid it leads to further division of the grid into smaller grids. As the region shown in the map goes smaller the bigger is the size of the grid. Each place will be stored in a hash table using key which is generated by a special hash function. The grids are referenced by their corresponding row and column number.

\section{Various Processes Involved:-}

$>$ Conversion of the voice i.e. analog wave to textual data via digital wave

Finding the places in the map

Giving detailed description of the places which comprises of :-

- The area of the place

- Paths available for user to the place

- Shortest distance between the user and the place

- And showing of other important places in the map near to the places searched.

Conversion of analog wave to digital wave can be facilitated by the use phonetics. There are phonemes in English language which is used to construct words. After the word is reframed 
from the phonetic dictionary available we compare the key of the place with that of the places stored in our hash table and obtain the grid number in which we can find that place and then locate it in that grid. Other detailed description about that place can be either easily saved in our database or can be calculated at that point of time. Area of the place, other important places near that place and all the available paths for the place from the position of the user can be easily stored in the database, whereas to calculate shortest distance from the user to the place we can use any of the available standards algorithms such as Flood-fill Algorithm or Djikistra's Algorithm.

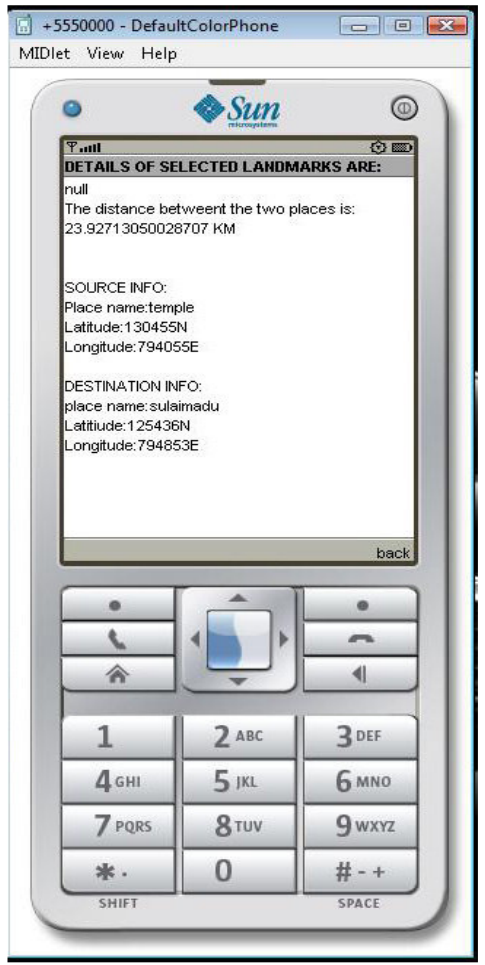

Fig-1 - The Distance and Co ordinates calculated using Offline Navigator

\section{Graphical User Interface (GUI):-}

The application will have the simple menu driven user interface. The user can select the input method i.e. Voice or text from the menu. Other options like zoom in, zoom out, details etc. can be selected from the menu. There will be a cursor which can be moved using navigation keys and any highlighted place can be clicked to get details. The possible paths can be highlighted with blue colour and shortest path by some prominent colour like red. The position of the user as well as the destination (if in the current screen) will be blinking. Other 
important places can be marked in some different colours. The option to update the maps will also be there in the menus.

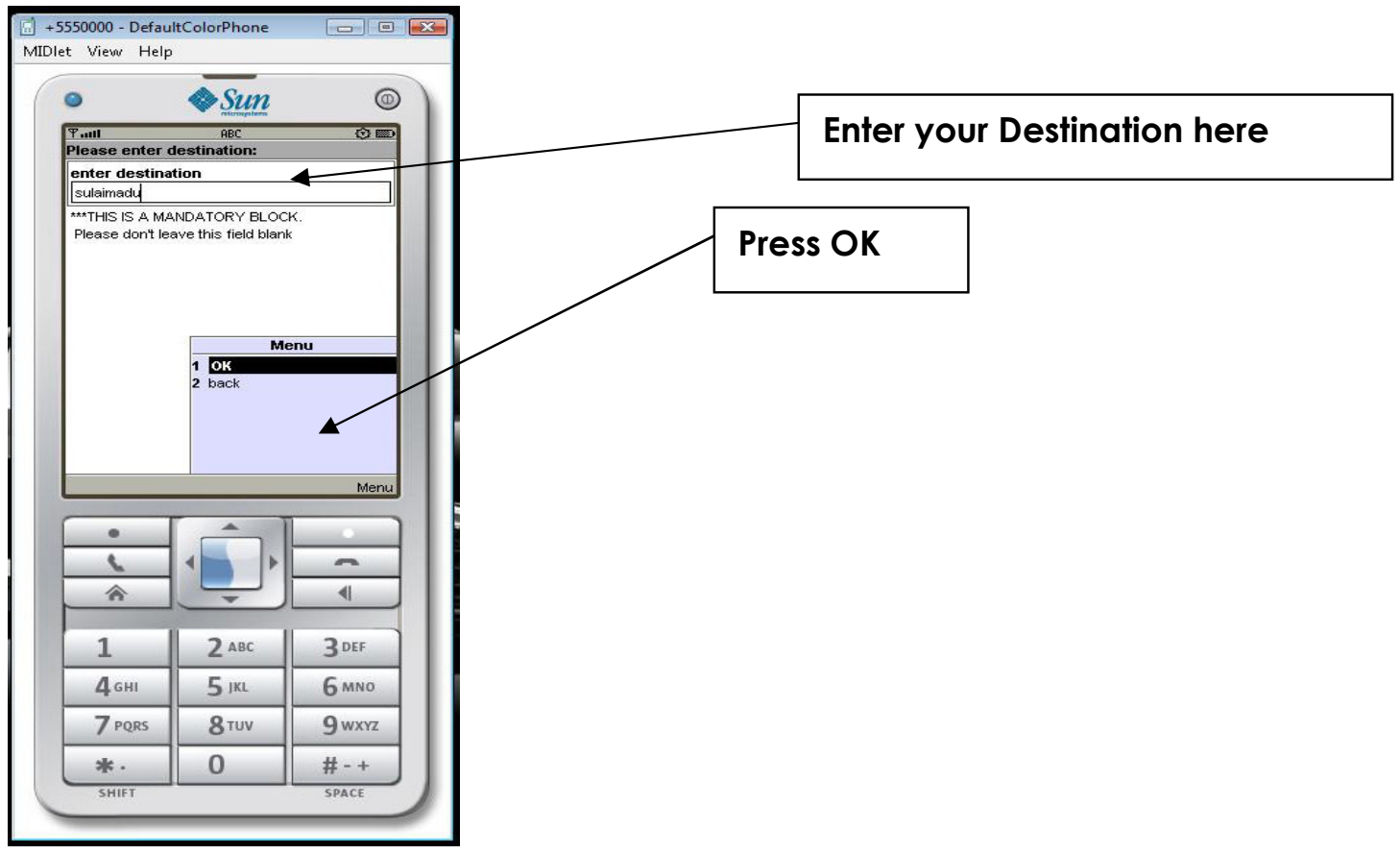

Fig 2- GUI Screenshot of Offline Navigator

\section{Update Mechanism:-}

If the user wishes to update the already installed maps or download a new map he has to connect the device to internet .The application will itself update the already installed maps and new maps can be installed by selecting the appropriate option on the site. Once downloaded on the device the updates will automatically update the database accordingly. Then the application can be restarted and will be ready to use. If any new features are added to in any of the updates the user will be informed by some pop-up window or balloon.

\section{MONETIZATION}

\section{Developing Cost of the Software}

The developing cost of our software is very low because most of the information and techniques used are already available. We just need to get the appropriate information and techniques together in a proper manner. The main expenditure will be on two things, buying the copyright of the maps and maintaining the web site from which the users can download 
the updates and maps of other cities. The copyright cost will depend upon the amount the institution charges for commercial use of the maps.

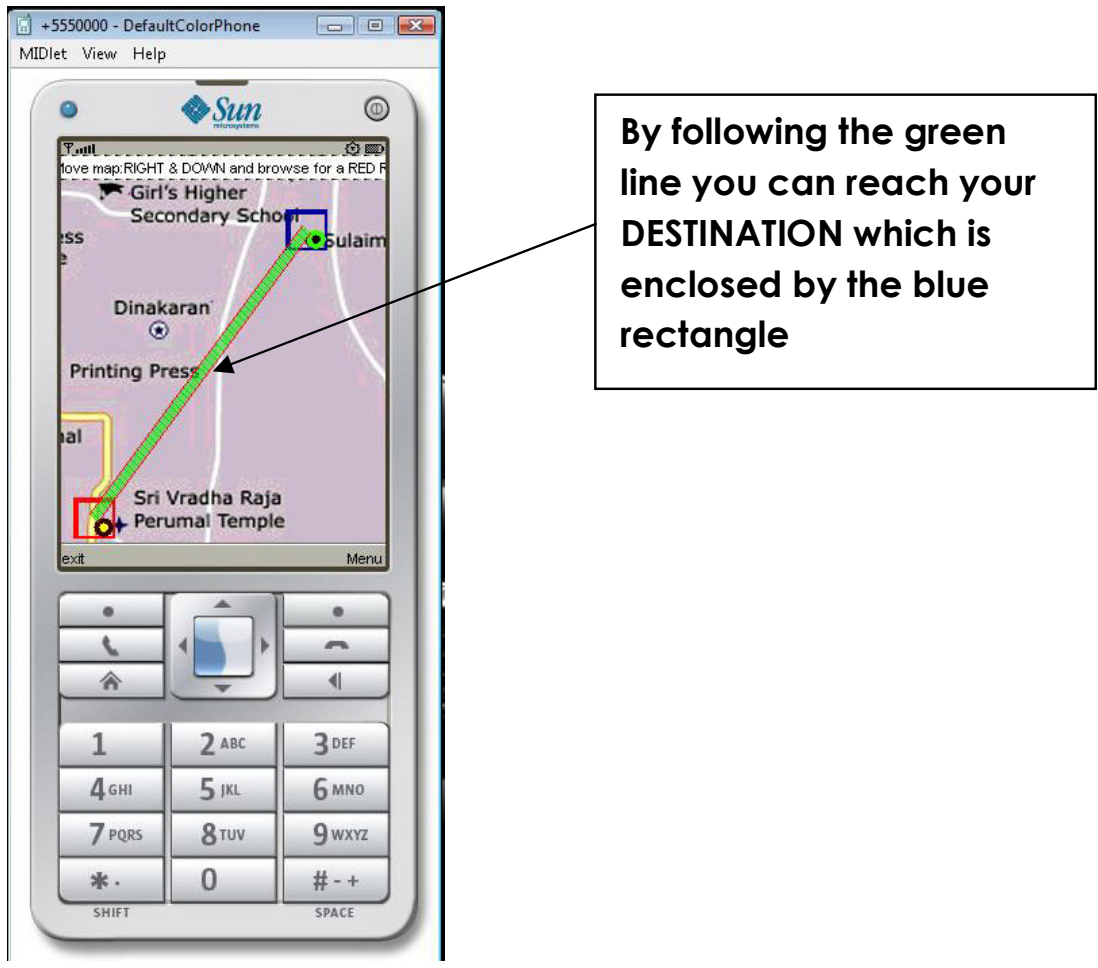

Fig 3 - Screenshot of Offline Navigator showing your source and destination and their connected path in the map.

\section{Cost - Effectiveness for the user or customer}

As the cost of development is low so naturally the cost of the service will also be very low for the end user. Not only will it be low cost but it will also save the consumer especially travellers the innumerable troubles faced by them while travelling. Moreover as this application requires very limited access to the internet so it further reduces the cost for the end user. The application created in java can be used on any java enabled handset thus putting aside the need of very costly mobile handsets and making the technology affordable and accessible for more and more people.

Thus we can safely conclude that our mobile application software will provide a better costeffectiveness to the users and also the cost of developing the software is quite low as compared to other online-mapping application or software which uses GPS or other expensive technologies. 
International Journal of Software Engineering \& Applications (IJSEA), Vol.1, No.2, April 2010

\section{SECURITY AND PRIVACY IMPLICATIONS}

As the mode of usage of this application is offline and the new packages/updates will be downloaded from a trusted site specifically meant for this purpose, there is virtually no chance that the security or the privacy of the user will be compromised. Thus the user can be completely assured that the information he is getting from the application is completely accurate and reliable. Moreover the offline mode eliminates any possibility of the mobile device or data stored in it getting corrupted or getting affected by any malicious software.

\section{POTENTIAL FOR EXTENSIBILITY}

By far the most prominent limitation of the application is that it requires a usable CDMA or GSM network. In absence of that the user will not be able to see his current location on the map. It is not completely independent of the internet; it has to be updated through it. The application is a totally platform independent so it will work on all kinds of platform for mobiles like Symbian, Linux, windows mobile etc. The services provided by the application can be further extended and services like travelling costs calculation and mobile tracking can provided in future. One of the major extensions could be linking of more than one device through the available GSM networks so that the location of more than one user can be displayed on all the connected devices. It can be used by local stores for advertising purposes as highest bidder can be displayed in the map.

\section{CONCLUSION}

Travellers all across the world often need a map for every place they visit and our product will be ready in every way in order to help them. This product is a boon to the people who are illiterate because of its voice recognition ability. Now they don't have to type the name of the places they wish to visit but they have to speak the name of the place and the navigator will find the place for them. Its quiet different from rest of the navigators due to it's use in offline mode. Now days various maps available are online and the people in the remote areas could not access them, either due to no internet or low speed of the network. Many of them even do not know how to use them but our navigator is easy to work with. It's independency on any internet work makes it's most desirable. There is a large amount database existing at the present time which is never accessible by a common man but our software will make them easily access these data just through a mobile. 


\section{BASIC WORKING PROTOTYPE OF THE SOFTWARE}

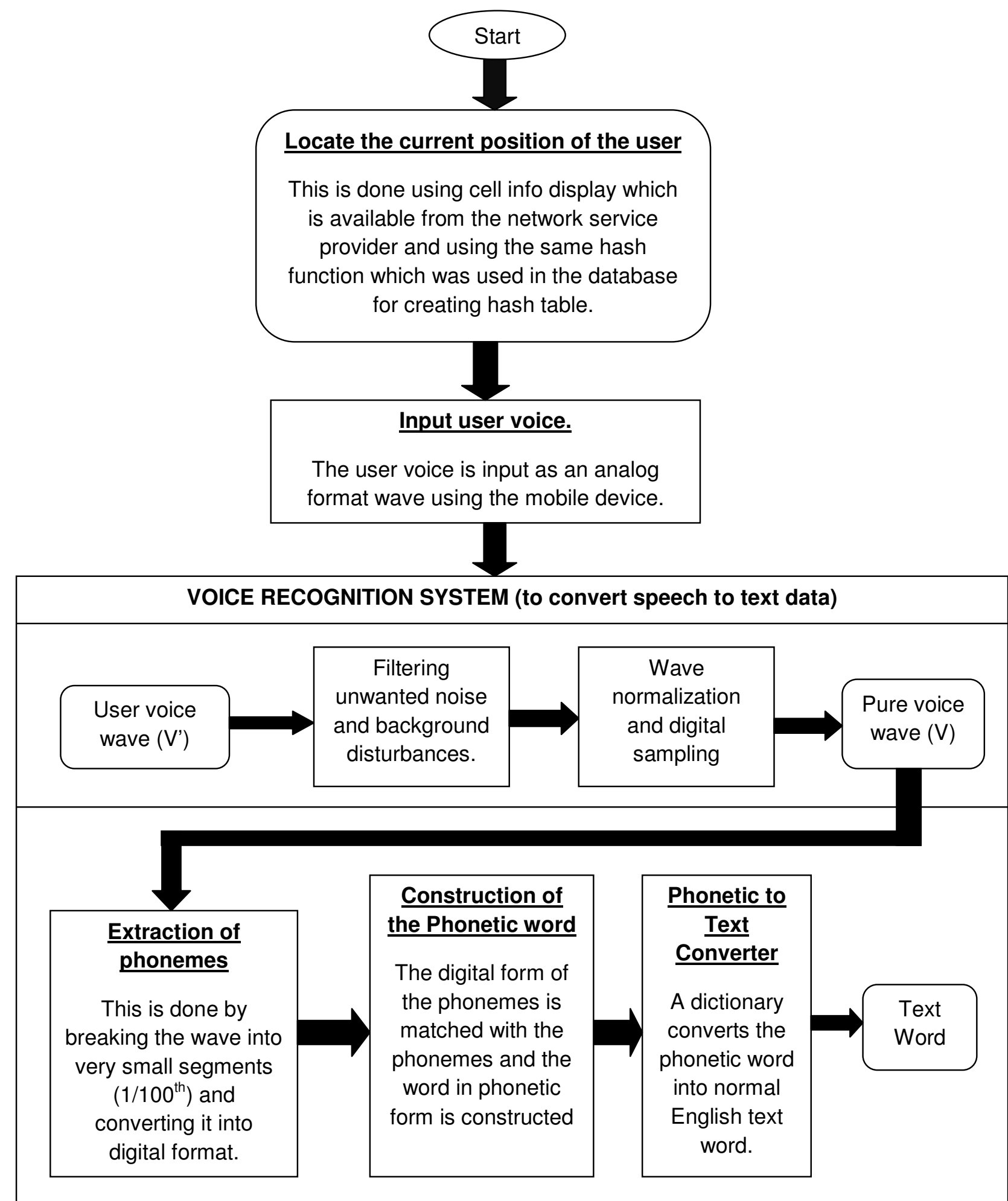




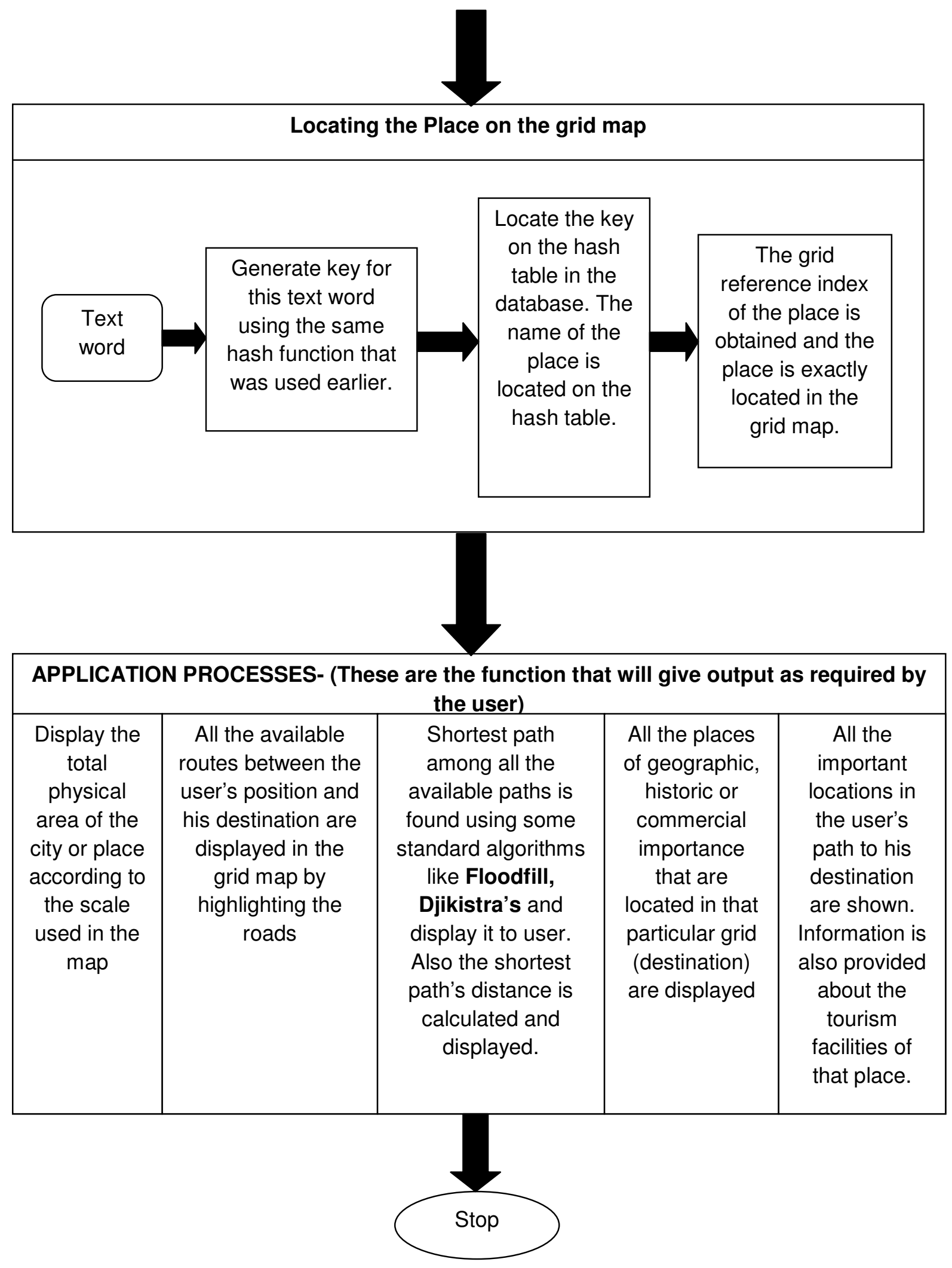


The portability of our software is immensely attractive as it needs no other device for its application rather it needs only a mobile which is java enabled. Now days many travellers, tourists need guide for travelling places which are not easily available to them always or available at higher at higher rates but this software will solve this problem easily as it will show the tourist places of all the cities and describe them precisely.

\section{ACKNOWLEDGEMENT}

We acknowledge the funding from Google, India (Bangalore) for helping us develop a working prototype of our software.

\section{REFERENCES}

1. Java 2 Micro Edition - The Complete Reference, Herbt Schidt, TMH Publication

2. Alternative Positioning Method Using GSM Signals - Associate Professor - Goh Pong Chai, The 2004 International Symposium on GNSS/GPS.

3. Catch this Speech Event - Recognition and Synthesis on Devices - Charles Hemphill, Steve Rondell, Conversay, www.conversay.com TS-5932

4. Wireless Messaging API for Java 2 Micro Edition Version 1.1, Sun Microsystems Documentation.

\section{ABOUT AUTHORS}

Prithumit Deb is a student pursuing B-Tech in Information Technology at School of Information Technology and Engineering at VIT University, Vellore, Tamilnadu, India. His research areas of interest are Web Services and Web Development, Mobile Application Development and Location Based Services. He has published papers at conferences like INCEB and was one of the finalists in Google Product prodigy Competition 2009 held at Google Bangalore.

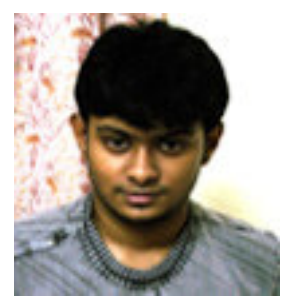

Nitin Singh is a student pursuing B-Tech in Information Technology (IT) at Vellore Institute of Technology University, Vellore Tamilnadu, India. His field of interests include J2ME mobile application development, Web development and Location based services. He has published papers at conferences like INCEB and was one of the finalists in Google Product prodigy Competition 2009 held at Google Bangalore.

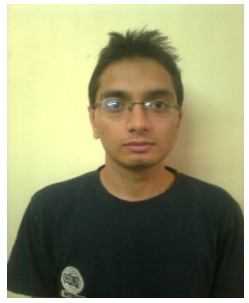


Saket Kumar is a student of B-Tech Information Technology at School of Information Technology and Engineering at VIT University, Vellore, Tamilnadu, India. His research areas of interest are Web Development, Mobile Application Development, Network Security and Robotics. He has published papers at conferences like INCEB, ICISA 2010 and was one of the finalists in Google Product prodigy Competition 2009 held at Google Bangalore.

Nitish Rai is a B.Tech student pursuing Information Technology (IT) at Vellore Institute of Technology University. His field of interests include J2ME mobile application development, Web development and Data Structures. He has published papers at conferences like ICISA 2010 and was one of the finalists in Google Product prodigy Competition 2009 held at Google Bangalore.
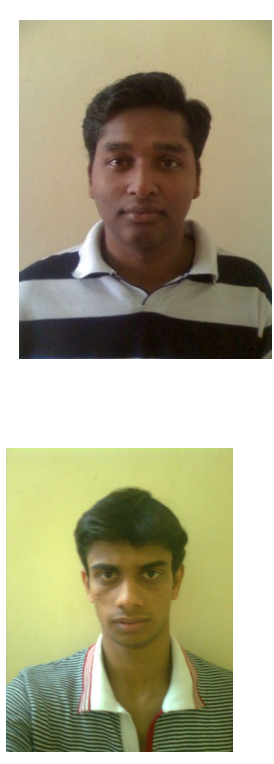

Dr. P. A. S. Naidu presently principal of D. B. Science College, Gondia (MS), India.He has been award as the Best Principal Award by RTM Nagpur University, Nagpur. His specialization is in Approximation Theory and Graph Theory. He did a sponsored Research Project sponsored by U.G.C., entitled - "APPLICATION OF GRAPH THEORY". He organized and attended several international conferences.

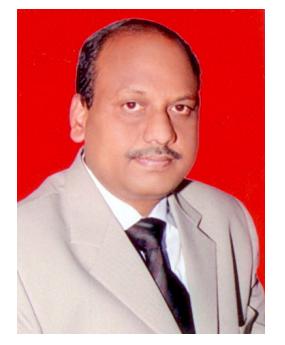

Dr.N.Ch.S.N.Iyengar is a Senior Professor at the School Of Computing Sciences at VIT University, Vellore, Tamilnadu India. He received M.Sc (Applied Mathematics) $\& \mathrm{PhD}$ from Regional Engineering College Warangal (Presently known as NIT Warangal).Kakatiya University, Andhra Pradesh, India, \& M.E. (Computer Science and Engineering) from Anna University, Chennai, India. His research interests include Fluid Dynamics (Porus Media), Agent based E-Business Applications, Data Privacy, Image Cryptography, Information security, Mobile Commerce and cryptography. He has authored several textbooks and had more than100 research

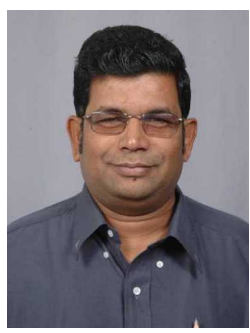
Publications in National, International Journals \& IEEE/ACM/WI Conferences. He is also Editorial Board member for many National and International Journals. He chaired many International conferences' and delivered invited, technical lectures along with keynote addresses besides being International programme committee member 